[Chem. Pharm. Bull.

36(12)4941-4950(1988) $\mid$

\title{
Supersaturation Mechanism of Drugs from Solid Dispersions with Enteric Coating Agents
}

\author{
Akihiko Hasegawa, ${ }^{* a .1)}$ Masahiro Taguchi, ${ }^{a}$ RIE Suzuki, ${ }^{a}$ \\ Tatsuhiko Miyata, ${ }^{a}$ Hiroshi Nakagawa, ${ }^{a}$ \\ and ISAO SUGImoto ${ }^{b}$
}

Pharmaceuticals Research Center, Kanebo Ltd., 1 1-5-90, Tomobuchi-cho, Miyakojima-ku, Osaka 534, Japan and Department of Pharmacy, Kitano Hospital, ${ }^{b}$ 13-3, Kamiyama-cho, Kita-ku, Osaka 534, Japan

(Received May 13, 1988)

\begin{abstract}
The crystallization behavior of drugs from supersaturated solutions containing carboxymethylethylcellulose (CMEC) was investigated to clarify the mechanism of supersaturation phenomena from solid dispersions with enteric coating agents in JPXI 2nd fluid (pH 6.8). Nifedipine, griseofulvin and spironolactone were used as drugs. The rate of crystallization of all drugs was remarkably inhibited by the presence of CMEC. It was found that the inhibition was not due to solubilization of the drugs by CMEC.

The crystallization kinetics from supersaturated solutions suggested that the process of crystal growth itself was directly inhibited. Physical properties of the crystallized mass were also investigated in the case of nifedipine. It was thought that the inhibitory effect of CMEC on the drug crystallization was due to the adsorption of CMEC at the solid-water interface at the stage in which a hydrophobic drug crystal surface was formed, and further drug molecules could not deposit easily on the crystal surface for the following reasons; (1) formation of a step or kink, which is necessary for the crystal growth, was inhibited by polymer adsorption, and (2) molecular diffusion to the crystal surface was inhibited by the adsorbed polymer.
\end{abstract}

Keywords_-nifedipine; griseofulvin; spironolactone; solid dispersion; supersaturation; crystallization; crystal growth

Chiou and Riegelman defined a "solid dispersion" as a dispersion of one or more active ingredients in an inert carrier or matrix in the solid state prepared by the melting (fusion), solvent, or melting-solvent method. ${ }^{2)}$

It is well known that many drugs in solid dispersions are present as a high-energy amorphous phase and a solid dispersion using a water-soluble carrier shows supersaturation of a poorly soluble drug when the system is added to an aqueous medium. ${ }^{3)}$ It can be considered that the dissolution of the drug takes place in the solid state prior to its exposure to the liquid medium. ${ }^{2)}$

A few investigators have examined the dissolution mechanism of solid dispersions. ${ }^{4)}$ However, quantitative analysis of the dissolution behavior in the amorphous state is quite difficult, since the amorphous state does not possess heat of fusion or solution, and this state transforms to the stable crystalline form easily during the experimental period.

We attempted to apply a solid dispersion technique using different types of enteric coating agents as inert carriers to control the absorption behavior of water-insoluble drugs. ${ }^{5}$ Most of the drugs in these solid dispersions were amorphous, and the dissolution of the solid dispersions was practically nil in JPXI 1st fluid (JP XI disintegration medium, pH 1.2). However, they dissolved rapidly in JP XI 2nd fluid (JP XI disintegration medium, pH 6.8) and showed supersaturation phenomena. We also investigated the crystallization behavior of nifedipine (NP) from supersaturated solutions containing various polymers, ${ }^{6}{ }^{6}$ since it was 
thought that supersaturation behavior might play an important roll in drug absorption.

In this report, NP, spironolactone (SL) and griseofulvin (GF) were used as model drugs, and supersaturation and crystallization of drugs were further investigated. The effect of polymer on the drug dissolution from solid dispersions with enteric coating agents is discussed.

\section{Experimental}

Materials - NP, SL, and GF were of JP XI grade. NP was pulverized to a fine powder. The particle size determined by an air permeability method was about $3 \mu \mathrm{m}$. Hydroxypropylmethylcellulose phthalate (HP-55, JP XI grade, Shin-Etsu Chemical Ind., Co., Ltd.), carboxymethylethylcellulose (CMEC. Freund Ind., Co., Ltd.), cellulose acetate phthalate (CAP, JP XI grade, Wako Pure Chemical Ind., Ltd.), and methacrylic acid -methacrylic acid methyl ester copolymer (Eudragit L, Rhöm Pharma) were used as received. Other chemicals were of reagent grade.

Preparation of NP Solid Dispersions - NP solid dispersions with enteric coating agents were prepared by the solvent method reported in the previous paper. ${ }^{5 a)}$

Dissolution Study of NP Solid Dispersions - A simple beaker-stirrer dissolution method as reported in the previous paper was employed. ${ }^{5 a}$ JP XI 2 nd fluid was used as the test medium. The test solution was filtered and assayed spectrophotometrically.

Effect of CMEC on the Crystallization of Drugs from Supersaturated Solutions - CMEC was used as an additive, and the effect of a polymer on the crystallization of drugs was investigated in the same manner as used in the dissolution test. The experiments were carried out in duplicate or triplicate.

Effect of the Presence of CMEC: CMEC $(150 \mathrm{mg})$ was dissolved in $500 \mathrm{ml}$ of JP XI 2nd fluid at $37 \mathrm{C}$. NP, SL and $\mathrm{GF}(1.25 \mathrm{~g})$ were dissolved in $50 \mathrm{ml}$ of $\mathrm{MeOH}, \mathrm{EtOH}$ and dimethylformamide, respectively. The drug solution $(2 \mathrm{ml})$ was added to the JPXI 2nd fluid containing CMEC. The rates of crystallization of drugs were measured by spectrophotometry.

Effect of CMEC Concentration: CMEC (5-150 mg) was dissolved in $500 \mathrm{ml}$ of JP XI 2nd fluid at $37 \mathrm{C}$. Drug solution $(4 \mathrm{ml}, 25 \mathrm{mg} / \mathrm{ml})$ was added to the test medium and the rates of crystallization were measured.

Effect of Seeding: CMEC $(150 \mathrm{mg})$ was dissolved at $37^{\circ} \mathrm{C}$ in $500 \mathrm{ml}$ of JP XI 2nd fluid, into which $0.1-1.0 \mathrm{~g}$ of NP fine powder (mean particle diameter, $3 \mu \mathrm{m}$ ) had been dispersed. Then, $4 \mathrm{ml}$ of NP methanolic solution $(25 \mathrm{mg} / \mathrm{ml})$ was added to the medium, and the change of NP concentration was measured by spectrophotometry.

Effect of pH: CMEC $(500 \mathrm{mg})$ was dissolved in $500 \mathrm{ml}$ of $0.005 \mathrm{~N} \mathrm{NaOH}$. Then, $0-200 \mathrm{ml}$ of $0.005 \mathrm{~N} \mathrm{HCl}$ was added to $150 \mathrm{ml}$ of CMEC solution and diluted with water to $500 \mathrm{ml}$. The $\mathrm{pH}$ of the solution was measured and $4 \mathrm{ml}$ of NP methanolic solution $(25 \mathrm{mg} / \mathrm{ml})$ was added. The amount of NP dissolved was measured by spectrophotometry at $4 \mathrm{~h}$ after the addition of NP solution.

Solubility of Drugs - In order to investigate the solubilizing effect of CMEC, $20 \mathrm{mg}$ of each drug was dispersed in $100 \mathrm{ml}$ of JPXI 2nd fluid containing $30 \mathrm{mg}$ of CMEC. A tightly capped flask containing sample solution was shaken horizontally in a water bath maintained at $37^{\circ} \mathrm{C}$ for $4 \mathrm{~d}$, then the solution was filtered. The drug concentration was measured.

Particle Size of Crystallized Drug — Mean particle diameter of crystallized drugs was measured with a particle size analyzer (PCA-11, System Science Co., Ltd.).

Membrane Permeability of Drugs - In order to identify the association of CMEC and the drugs, membrane permeability of drugs in the presence of CMEC was investigated using the equilibrium dialysis technique and a diffusion cell. The membrane used in these studies was seamless cellulose tubing (Visking Company).

Equilibrium Dialysis: CMEC and drugs were dissolved in JP XI 2nd fluid. The concentrations of CMEC and drugs were 300 and $10 \mu \mathrm{g} / \mathrm{ml}$, respectively. Ten milliliters of the solution was sealed in the cellulose tubing and the tubing was immersed in $100 \mathrm{ml}$ of JPXI 2 nd fluid at $37^{\circ} \mathrm{C}$. The concentration of the drug in each compartment was measured after $3 \mathrm{~d}$.

Diffusion Cell: NP was used as a model drug. The glass cell consisted of donor and receptor compartments and the membrane (available area $=7.07 \mathrm{~cm}^{2}$ ) placed between them. Eighty milliliters of JP XI 2nd fluid was poured into each compartment. CMEC (24.mg) was dissolved in the donor solution, and $320 \mu \mathrm{l}$ of methanolic solution equivalent to $8 \mathrm{mg}$ of NP was added to the donor cell. The content of each compartment was stirred with a magnetic stirrer, and the permeation rate of NP was measured.

\section{Results and Discussion}

\section{Dissolution Behavior of NP Solid Dispersions}

Effect of the Amount of Sample Added on the Dissolution Behavior-Figure 1 shows the dissolution behavior of NP from solid dispersions (NP : polymer =1:3) in JP XI 2nd fluid. 


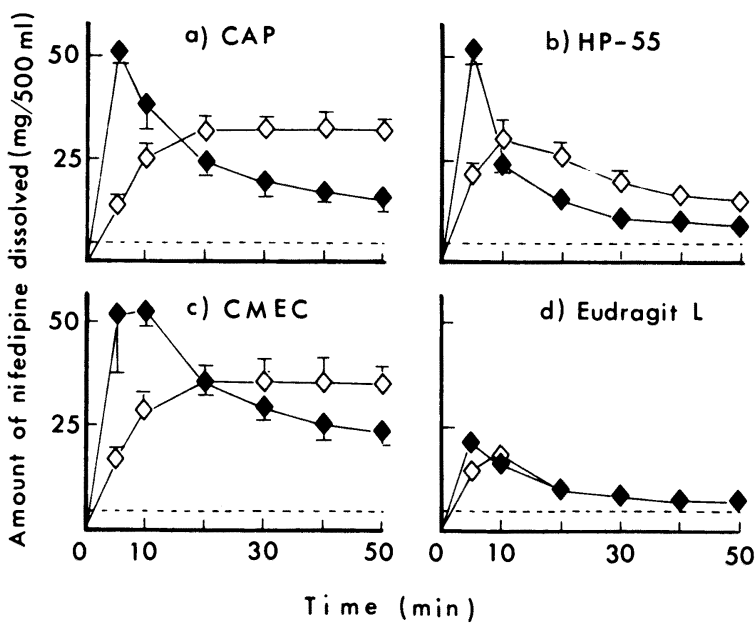

Fig. 1. Dissolution Behavior of NP from Solid Dispersions (NP : Polymer $=1: 3$ ) in JP XI 2nd Fluid

The amount of sample added: NP equivalent to 50 $(\diamond)$ or $150 \mathrm{mg}(\diamond)$. Each point represents the average \pm S.D. The dotted line shows the solubility of NP in JP XI 2nd fluid.

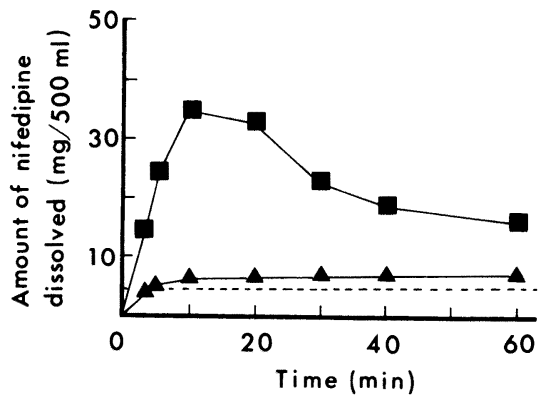

Fig. 2. Dissolution Behavior of NP from Eudragit L Solid Dispersions in JPXI 2nd Fluid Containing $150 \mathrm{mg}$ of CMEC

NP: Eudragit $L \Delta, 1: 1 ; \mathbf{\square}, 1: 3$. The dotted line shows the solubility of NP in JP XI 2nd fluid.

In this case, the sample equivalent to 50 or $150 \mathrm{mg}$ of NP was dispersed in $500 \mathrm{ml}$ of dissolution medium at $37 \mathrm{C}$. In the case of Eudragit L solid dispersion, the sample amount did not affect the dissolution behavior of NP, and distinct supersaturation was not observed. However, the dissolution behavior was remarkably affected by the amount of sample added in the cases of cellulose polymers. That is, the greater the amount of sample, the faster the rate of crystallization.

As the formation of crystal nuclei and the growth of crystals are comparatively complex phenomena, the theoretical treatment of growth kinetics has not yet been fully developed. This process should be considered in detail to clarify the mechanism of supersaturation.

It was estimated that the remarkable supersaturation of NP from the solid dispersions using cellulose polymers such as CMEC, CAP, or HP-55 was due to the inhibitory effect of these polymers on the crystallization of NP. ${ }^{6)}$ Because of its high inhibitory effect, CMEC was selected and the crystallization behavior of NP from supersaturated solutions was further investigated in the presence of CMEC.

Effect of CMEC on the Dissolution Behavior of Eudragit L Solid Dispersion-Eudragit L solid dispersions were dispersed in JPXI 2nd fluid containing $150 \mathrm{mg}$ of CMEC, and the dissolution behavior of NP was investigated to confirm the inhibitory effect of CMEC on the recrystallization of NP (Fig. 2).

NP dissolved rapidly from the solid dispersion (NP: Eudragit $\mathrm{L}=1: 3$ ) and showed supersaturation. However, remarkable supersaturation was not observed in the case of solid dispersion (NP: Eudragit $\mathrm{L}=1: 1$ ) even in the presence of CMEC. It was found in the previous paper $^{5 a)}$ that NP was present in its amorphous form in $(1: 3)$ solid dispersion, but was present in its crystalline form in $(1: 1)$ solid dispersion by X-ray diffraction analysis. It was thought that marked supersaturation from a solid dispersion was only maintained when the following two requirements were satisfied at the same time, that is, a) the drug is in an amorphous state in the solid dispersion, and b) a substance is present which inhibits the crystallization of the drug from the supersaturated solution. 


\section{Inhibition Mechanism of Crystal Growth by CMEC}

Crystallization Behavior of Drugs from Supersaturated Solution_- In order to estimate the inhibitory effect of CMEC on the crystallization of drugs from supersaturated solution, NP, SL and GF were used as model drugs. Organic solution equivalent to $50 \mathrm{mg}$ of each drug was added to $500 \mathrm{ml}$ of JPXI 2nd fluid containing $150 \mathrm{mg}$ of CMEC. Figure 3 shows the crystallization behavior of each drug with or withour CMEC.

CMEC inhibited the crystallization of the drugs from supersaturated solution. As it was thought that the solubilization of drugs by CMEC might affect the inhibition phenomena, the solubility value of each drug in JP XI 2nd fluid containing CMEC was investigated. The solubility ratios of NP, GF, and SL with and without CMEC were 0.94, 1.09 and 0.93, respectively. It is unlikely that the supersaturation phenomena are due to the solubilizing effect of CMEC.

Additionally, the chemical interaction between CMEC and each drug was investigated using the dialysis technique. There was no evidence of CMEC-drug complex formation in an equilibrium dialysis study using cellulose tubing. That is, the drug concentration ratio of donor and receptor solution was about 1 , even when CMEC was present in the donor solution. The permeability characteristics were further investigated using NP. That is, the release profile of NP through a cellulose membrane from its solution $(100 \mu \mathrm{g} / \mathrm{ml})$ in JP XI $2 \mathrm{nd}$ fluid containing CMEC $(300 \mu \mathrm{g} / \mathrm{ml})$ was determined by using a diffusion cell. NP was released linearly through the membrane. The release rate was $4.6 \mu \mathrm{g} / \mathrm{min}$. The concentration of NP in the donor solution was maintained at about $100 \mu \mathrm{g} / \mathrm{ml}$. The permeability was also investigated without CMEC. In this case, the donor concentration of NP was $10 \mu \mathrm{g} / \mathrm{ml}$, since a supersaturated solution could not be obtained. The release rate through a cellulose membrane from this solution (NP $10 \mu \mathrm{g} / \mathrm{ml}$ ) in JP XI 2nd fluid without CMEC was $\theta .46 \mu \mathrm{g} / \mathrm{min}$. These results showed that the release rate was only a function of NP concentration in the donor solution, and the presence of CMEC did not affect the release rate of NP. From these results, it was concluded that the supersaturation phenomenon was not due to complex formation between CMEC and the drug.

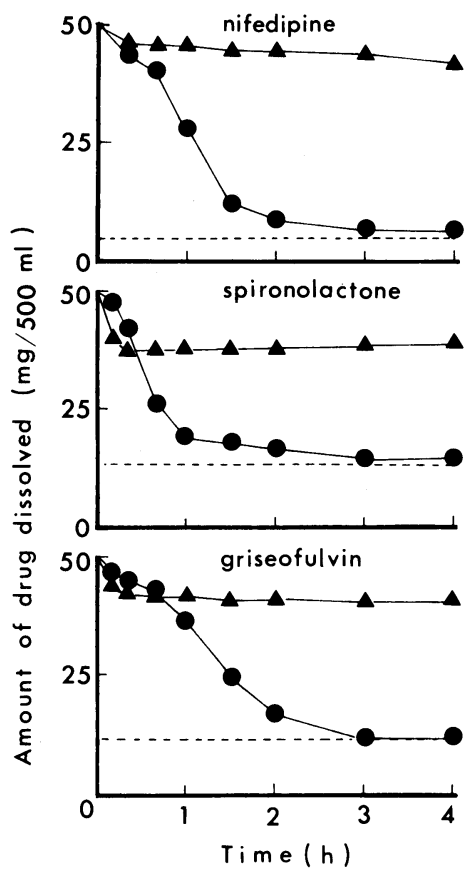

Fig. 3. Recrystallization Behavior of the Drug in JPXI 2nd Fluid Containing $150 \mathrm{mg}$ of CMEC

A, with CMEC; $\bullet$, without CMEC. The dotted line shows the solubility of the drug in JP XI 2nd fluid. 
Crystallization Kinetics of Drugs from Supersaturated Solution - The kinetics of crystal growth from supersaturated solution can be generally represented by a simple power-law equation of the form ${ }^{7}$ :

$$
R=K\left(C-C_{\mathrm{e}}\right)^{n}
$$

where $R$ is the crystal growth rate, $K$ is the growth rate coefficient, $C$ and $C_{\mathrm{e}}$ are the bulk solution concentration at time $t$ and the equilibrium (saturated) concentration, and $n$ is the growth order.

Kawashima et al. ${ }^{81}$ evaluated the crystallization kinetics as follows. The mass of crystal $W$ is represented by Eq. 2 .

$$
W=\left(C_{\mathrm{i}}-C\right) \mathrm{V}
$$

where $C_{\mathrm{i}}$ is the initial bulk concentration and $V$ is the volume of the solution. The mass growth rate Eq. 3 is derived by differentiating Eq. 2 with respect to time $t$.

$$
R=\mathrm{d} W / \mathrm{d} t=\mathrm{d}\left(C_{\mathrm{i}}-C\right) V / \mathrm{d} t=-\mathrm{d}\left(C-C_{\mathrm{e}}\right) V / \mathrm{d} t
$$

From Eq. 1 and Eq. 3:

$$
-\mathrm{d}\left(C-C_{\mathrm{e}}\right) V / \mathrm{d} t=K\left(C-C_{\mathrm{e}}\right)^{n}
$$

When $n$ is equal to one, showing that the kinetics was corresponds to diffusion-controlled crystal growth, the decrease of supersaturation with time during crystallization followed an exponential decay as expressed by:

$$
\ln \left(C-C_{\mathrm{e}}\right)=-K t / V+E
$$

where $E$ represents the supersaturation at the onset of precipitation.

Figure 4 shows the crystallization of drugs from JPXI 2nd fluid containing various concentrations of CMEC. The drug $(100 \mathrm{mg})$ was added as an organic solution $(25 \mathrm{mg} / \mathrm{ml})$, and the logarithm of $\left(C-C_{\mathrm{e}}\right)$ was plotted versus time. The solubility value of each drug in JP XI 2nd fluid was used as $C_{\mathrm{e}}$.

In the case without CMEC, the change in bulk solution concentration followed firstorder kinetics for all drugs. However, the linear relationship between $\ln \left(C-C_{\mathrm{e}}\right)$ and $t$ was not observed in the presence of CMEC, showing apparent non-diffusion-controlled crystal growth. That is, a process other than the transfer of drug molecules in the diffusion layer

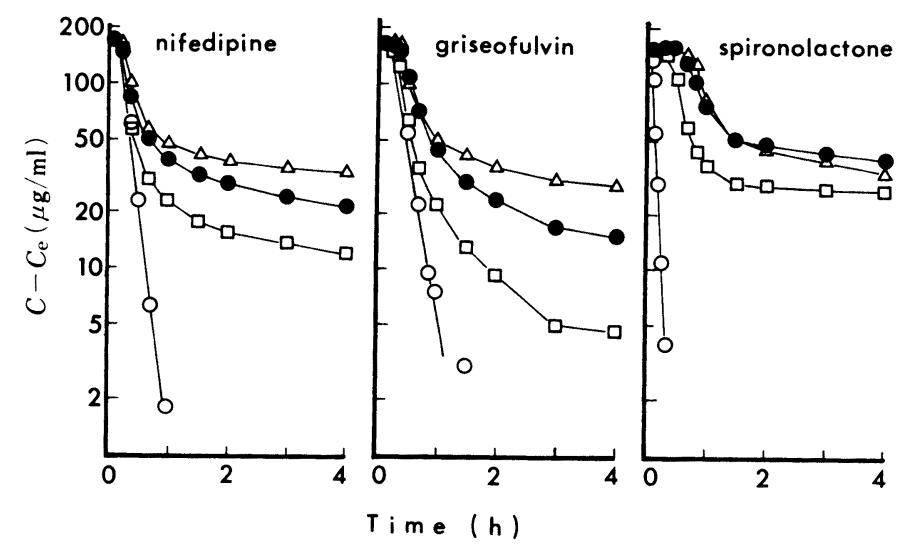

Fig. 4. Effect of CMEC Concentration on the Recrystallization of the Drug in JPXI 2nd Fluid

CMEC concentration (mg/500 ml): $\square, 5 ; \bullet, 30 ; \triangle, 150 ; \bigcirc$, without CMEC. 


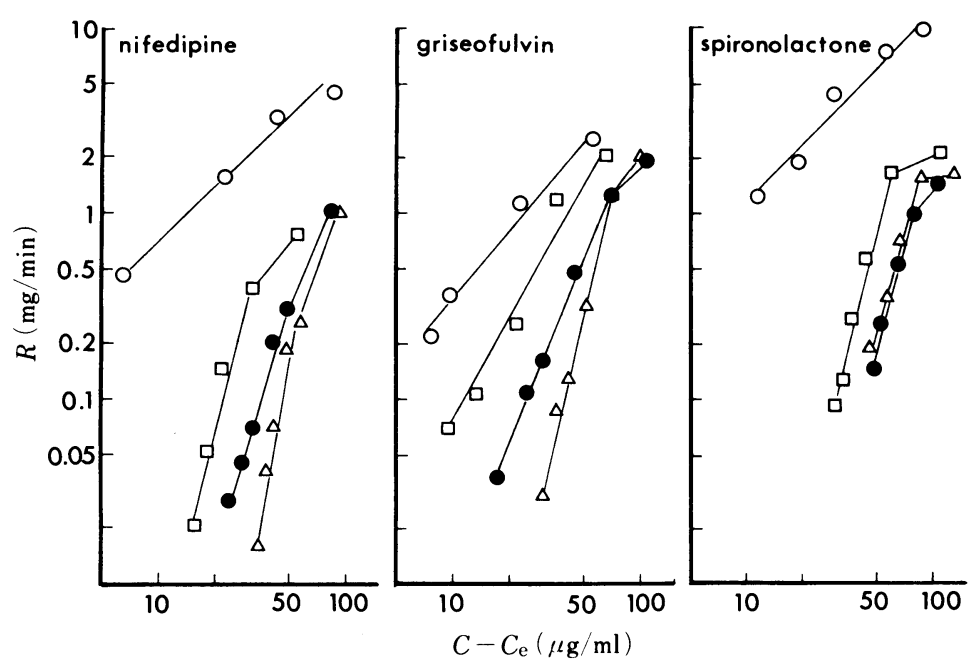

Fig. 5. $\log R$ versus $\log \left(C-C_{\mathrm{e}}\right)$ at Various CMEC Concentrations for the Crystallization of the Drug in JPXI 2nd Fluid

CMEC concentration $(\mathrm{mg} / 500 \mathrm{ml}): \square, 5 ; \bullet, 30 ; \triangle, 150 ; \bigcirc$, without CMEC.

might be rate-determining. The mass growth rate at time $t$ was calculated. A log-log plot of growth rate $R$ versus supersaturation $\left(C-C_{\mathrm{e}}\right)$ is expected to be linear from Eq. 1, and $n$ could be determined readily from the slope (Fig. 5).

In the case without polymer, the slope was nearly equal to one for all the drugs. In the presence of CMEC, $R$ is biphasic, and the resulting $n$ at low supersaturation is larger than that at high supersaturation. The large value of $n$ in this case means that the growth rate decreases extremely rapidly with a small decrease in supersaturation. For instance, supersaturation of $\mathrm{NP}$ was maintained even after $6 \mathrm{~d}$ in the case when $150 \mathrm{mg}$ of CMEC was added. That is, the concentrations of NP after $4 \mathrm{~h}$ and $6 \mathrm{~d}$ were 43.3 and $34.5 \mu \mathrm{g} / \mathrm{ml}$, respectively.

Microscopic Observation of Crystallized Drugs_-Figure 6 shows the results of microscopic observation of crystallized drugs in JP XI 2nd fluid containing CMEC at $4 \mathrm{~h}$ after the addition of a drug solution.

The shape of drug crystals changed remarkably with increase of CMEC concentration. That is, a finely divided spherical mass was obtained at higher CMEC concentration.

NP was selected and the effect of CMEC on the crystallization of the drug was further investigated.

Effect of Seeding — The crystallization process can be divided into nucleation and crystal growth. The nucleation rate of substances from supersaturation is given by the Arrhenius equation, and the rate is affected by the frequency factor, temperature, degree of supersaturation, and interfacial tension. ${ }^{9)}$ Therefore, it was presumed that the presence of CMEC altered the nucleation rate of $d r u g$. This change may affect the mode of crystallization. Then, various amounts of NP crystals (mean particle diameter, $3 \mu \mathrm{m}$ ) were added as seeds instead of nuclei, since it is difficult to follow the nucleation process. The effect of seeding on the crystallization of NP in the presence of CMEC was investigated (Fig. 7).

The pattern of crystallization was biphasic under all experimental conditions. The concentration of NP decreased rapidly in the early phase, and the rate of crystallization decreased later. The rate of crystallization in the early phase increased with increase of the amount of seeding crystals. However, the slopes of the later phase were similar and the calculated value of $(n)$ from the slope was more than one in each case, showing apparent nondiffusion-controlled crystallization. When CMEC was not present, an extremely rapid 
( A)
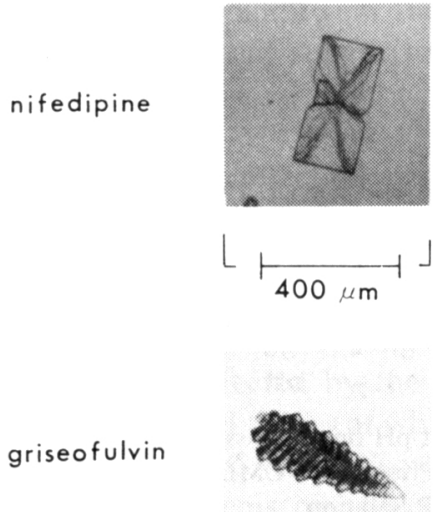

griseofulvin
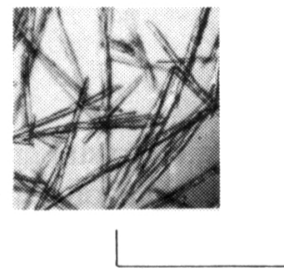

(B)
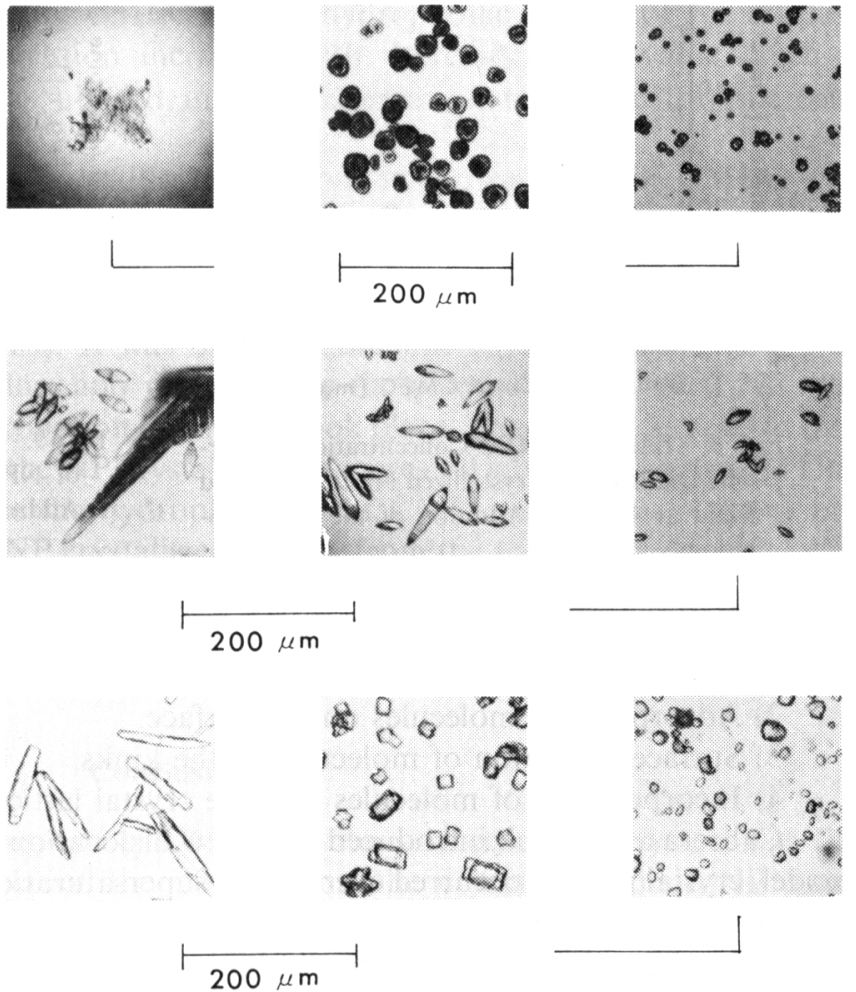

Fig. 6. Microscopic Observations of Crystallized Drug in JPXI 2nd Fluid Containing Various Concentrations of CMEC at $4 \mathrm{~h}$ after Addition of the Drug Solution

CMEC concentration $(\mathrm{mg} / 500 \mathrm{ml})$ : (A), 0; (B), 5; (C), 30; (D), 150 .

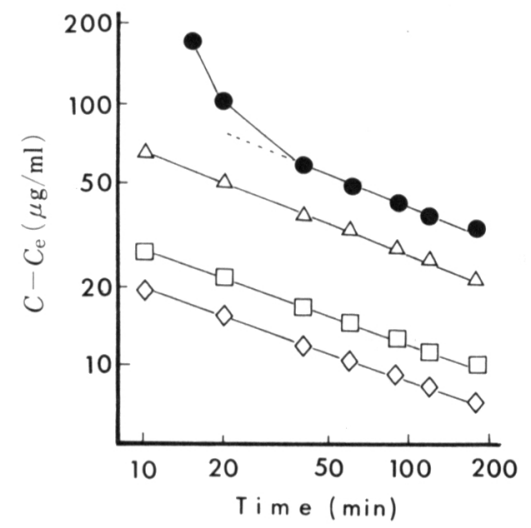

Fig. 7. $\log \left(C-C_{\mathrm{e}}\right)$ versus $\log t$ at Various Amounts of Seed Crystals for the Crystallization of NP

The amount of seed crystals $(\mathrm{g})$ $0.5 ; \diamond, 1.0$.

decrease of the concentration of NP to the solubility value was observed under each seeding condition. Thus, it was thought that the nucleation process was not the main factor controlling the apparent non-diffusion-controlled crystallization, but that the crystal growth itself was influenced by the presence of CMEC.

Particle Diameter of NP Crystallized from Supersaturated Solution-The following steps have been distinguished by Konak ${ }^{10)}$ for crystal growth from aqueous solution. 


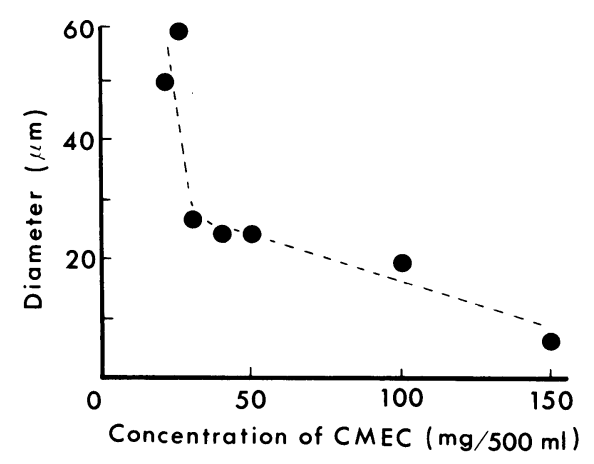

Fig. 8. Effect of CMEC Concentration on Particle Diameter of Crystallized NP in JP XI 2nd Fluid at $4 \mathrm{~h}$ after Addition of the Drug Solution

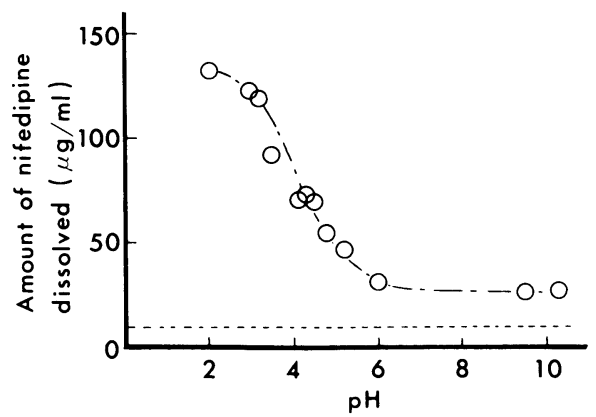

Fig. 9. Effect of $\mathrm{pH}$ on the Dissolved Amount of NP in the Presence of CMEC at $4 \mathrm{~h}$ after Addition of NP Solution

1) Transfer of molecules from the bulk solution to the immediate vicinity of the crystals by diffusion.

2) Adsorption of molecules on the surface.

3) Surface migration of molecules to the kinks.

4) Incorporation of molecules into the crystal lattices.

Cabrera and Frank introduced the screw dislocation model into crystal growth. ${ }^{11)}$ In this model, crystal growth occurred even at low supersaturation, since kinks or steps necessary for the crystal growth, were present continuously on the crystal surface. Thus, crystal growth essentially depends on the existence of kinks. Easy growth is guaranteed if kinks are always present. The presence of CMEC might affect each of the above-mentioned steps of the crystal growth process. Then, methanolic solution $(4 \mathrm{ml})$ equivalent to $100 \mathrm{mg}$ of NP was added to JP XI 2nd fluid containing various amounts of CMEC, and the effect of CMEC concentration on the particle diameter of crystallized NP was investigated. Figure 8 shows the particle diameter of crystallized NP at various CMEC concentrations at $4 \mathrm{~h}$ after the addition of NP.

The particle size decreased with increase of CMEC concentration. Additionally, it was observed that the particle size even in the initial stage of crystallization $(20$ min after the addition) was essentially the same as that of the later stage. These results showed that the process of crystal growth was inhibited by the presence of CMEC.

The crystallized mass was obtained by filtration and the properties of the mass were investigated. An X-ray diffraction study revealed that the crystallized substance had the same crystal structure as NP itself even when a large amount of CMEC (150 mg) was present. This crystallized mass did not show the supersaturation phenomenon, even when an excess amount of the mass was added to JPXI 2nd fluid with or without CMEC. These results showed that the formation of crystal structure was not directly inhibited. The NP content of the crystallized mass (150 mg CMEC) was $89.4 \pm 0.9 \%$. Thus, it was thought that the inhibitory effect of CMEC on the crystallization of NP was due to the adsorption or orientation of the polymer at the solid-water interface at the stage in which the hydrophobic NP crystal surface was formed. The adsorption of polymer on a hydrophobic surface was well discussed in a study on the stabilization of suspensions. ${ }^{12)}$ Three parameters describe completely the adsorption of a polymer from solution: the amount of adsorption per unit area of surface; the fraction of segments in contact with the surface; and the distribution of segments in the vicinity of the surface. ${ }^{13)}$

Effect of pH of the Test Medium-The hydrophobic character of an anionic polymer such as CMEC in solution varies with the $\mathrm{pH}$ value, due to the dissociation of carboxylic 
groups. That is, the hydrophobicity of CMEC increases with decrease of $\mathrm{pH}$ value. In this case, it was thought that the affinity of CMEC for the hydrophobic surface of NP crystals formed from the supersaturated solution increased with decrease of $\mathrm{pH}$ value. Such a phenomenon is well known, and is utilized in the coacervation technique for microencapsulation.

Then, the effect of $\mathrm{pH}$ on the crystallization of NP in the presence of CMEC was investigated. Figure 9 shows the effect of $\mathrm{pH}$ on the dissolved amount of NP at $4 \mathrm{~h}$ after the addition of NP.

In this experiment, $100 \mathrm{mg}$ of NP in methanolic solution was added to $500 \mathrm{ml}$ of test medium containing $150 \mathrm{mg}$ of CMEC. It was observed that the crystallization of NP was remarkably affected by the $\mathrm{pH}$ value, and in particular the amount of dissolved NP was increased at $\mathrm{pH}$ values at which protonation of CMEC took place. Thus, it was suggested that the amount of CMEC adsorbed on the NP crystal surface increased with decreasing $\mathrm{pH}$. This assumption seems plausible because the hydrophobicity of CMEC is increased in this $\mathrm{pH}$ range. In the case without CMEC, NP crystallized rapidly from the solution to the solubility value even in the low $\mathrm{pH}$ range. It was confirmed that the hydrophilic-hydrophobic property of the polymer affected the crystallization of NP and that there was a physical interaction between the polymer and NP in the solid state.

\section{Conclusion}

It was suggested that the adsorption of CMEC on the surface of NP crystals was the main factor in the inhibitory effect of the polymer on the crystallization of NP from supersaturated solution. However, the change of apparent diffusion layer thickness did not explain the phenomenon that the crystal growth was non-diffusion controlled. It was thought that the mechanism of crystal growth inhibition was due to difficulties of molecular deposition for the following reason(s) (1) and/or (2).

(1) Lack of steps or kinks on the crystal surface. A crystal surface with fewer steps or kinks might be formed by polymer adsorption. The drug molecules coluld not deposit easily on such a surface. "Bunching" was defined as an effect of impurities on the crystal growth. ${ }^{14}$ Small amounts of impurity are adsorbed on the crystal surface, and exert a protective effect, reducting the velocity with which the step travels. The size and shape of crystallized substance were different from those in the absence of impurity. These considerations seem compatible with the results of microscopy.

(2) Inhibition of molecular diffusion to the crystal surface by polymer adsorbed on the crystal surface. Chemical interaction of NP and polymer was not observed in dialysis and solubility studies. If highly concentrated polymer were present in the immediate vicinity of the crystal surface, it is possible that NP would interact with CMEC in this area, and this interaction might affect the crystallization behavior.

\section{References and Notes}

1) Present address: Institute of Pharma Research, Development and Medical Science, Nihon Schering K.K., 6-64, Nishimiyahara 2-chome, Yodogawa-ku, Osaka 532, Japan.

2) W. L. Chiou and S.: Riegelman, J. Pharm. Sci., 66, 1281 (1971).

3) T. Arita, The Pharmaceuticals Monthly, 21, 2737 (1979).

4) A. A. Simonelli, S. C. Mehta, and W. I. Higuchi, J. Pharm. Sci., 58, 538 (1969); H. Sekikawa, M. Nakano, and T. Arita, Chem. Pharm. Bull., 27, 1223 (1979); K. Takayama, N. Nambu, and T. Nagai, ibid., 28, 3304 (1980).

5) a) A. Hasegawa, H. Nakagawa, and I. Sugimoto, Yakugaku Zasshi, 104, 485 (1984); b) A. Hasegawa, R. Kawamura, H. Nakagawa, and I. Sugimoto, Chem. Pharm. Bull., 33, 3429 (1985).

6) A. Hasegawa, R. Kawamura, H. Nakagawa, and I. Sugimoto, Yakugaku Zasshi, 105, 586 (1985). 
7) J. S. Wey and R. Jagannathan, AIChE J., 28, 697 (1982).

8) Y. Kawashima, M. Okumura, and H. Takenaka, Powder Technol., 39, 41 (1984).

9) M. Okada, "Bunsisei-Kesshou," Saiwaishobo, Tokyo, 1975, p. 40.

10) A. R. Konak, Chem. Eng. Sci., 29, 1537 (1974).

11) N. Cabrera and F. C. Frank, Nature (London), 163, 398 (1949).

12) S. L. Law and J. B. Kayes, Int. J. Pharmaceut., 15, 251 (1983).

13) I. W. Kellaway and N. M. Najib, Int. J. Pharm. Technol. Prod. Manuf., 4, 41 (1983).

14) F. C. Frank, Growth and Perfection of Crystal, Proceeding of an International Conference on Crystal Growth, Cooperation, 1958, p. 411. 